\title{
Inpainting For Image Restoration
}

\author{
Chaitali P. Sathe ${ }^{1}$, Dr.Shubhalaxmi Hingway ${ }^{2}$,Sheeja Suresh $^{3}$ \\ ${ }^{I}$ (M.Tech, Departmentof Electronics and Telecommunication, G.H.Raisoni Institute of Engineering and \\ Technology ForWomen .Nagpur -India) \\ ${ }^{2}$ (Professor,Departmentof Electronics and Telecommunication, G.H.Raisoni Institute of Engineering and \\ Technology ForWomen .Nagpur-India) \\ ${ }^{3}$ (Asst. Professor,Departmentof Electronics and Telecommunication, G.H.Raisoni Institute of Engineering and \\ Technology ForWomen .Nagpur-India)
}

\begin{abstract}
This paper presents comparision between 2 ways of image inpainting for image restoration.A rough version of the input image is inpainted CDD inpainting technique and by TV inpainting technique. Image is usually statistically corrupted with noise , hence removal of the noise is another necessary objective of this paper. These ways are going to be applied to grey scale and RGB pictures. Compared to existing approaches, some enhancements are done. The inpainting of a rough version of the input image permits to scale back the machine complications, to be less vulnerable to noise and to figure with the dominant orientations of image structures. Experimental results on completely different pictures can show the effectiveness of the 2 ways.
\end{abstract}

Keywords: -Inpainting, CDD -curvature driven diffusion, TV -total variation

\section{INTRODUCTION}

For inpainting a broken image or associate ancient painting with missing regions is to guess and fill within the lost image data in such an identical manner that the restored image or painting appears as natural as its original version.

Applications of digital inpainting are:

(a) Restoration of ancient paintings for conservation functions

(b) Restoring aged or broken images and films

(c) Object removal and text removal in pictures for computer graphics

(d) Digital zooming and edge-based image cryptography

Mathematically, what makes the inpainting drawback thus difficult is that the quality of image functions.In contrast to several ancient interpolation or boundary worth issues, the target image functions to be inpainted usually lie outside the Sobolev class. structure complexities of image functions force researchers to develop inpainting schemes targeted at specific categories of pictures. As a result, these inpainting models are of low levels. The last word goal, of course, as within the blueprint of vision and computing, is eventually to be ready to mix and integrate all the low-level inpainting parts into a perfect program which will well approximate human inpainters.

Image inpainting is that the method of filling in missing components of broken pictures supported data gathered from close areas. Additionally to issues of image restoration, inpainting can even be utilized in wireless transmission and compression applications. during this project, we'll developed associate automatic digital in painting system that allows the user to settle on between 2 complementary approaches. The primary relies on the answer of partial equation of isophote intensity to reliever missing parts within the region into account, whereas the second relies on texture inpainting. The filling-in method is mechanically worn out regions containing utterly completely different structures, textures, and close backgrounds.

\section{RELATED WORK}

Some previous work worn out this field includes of a framework for examplar-based inpainting. It consists in acting 1st the inpainting on a rough version of the input image. A hierarchal super-resolution algorithmic program is then wont to recover details on the missing areas this was projected by O.Ebdelli, M.Guillemot, LeMeur. associate another algorithmic program was introduced by Bertalmio, M., Sapiro, G., Caselles, V., Ballester, C, for digital inpainting of still pictures that tries to copy the fundamental techniques employed by skilled restorators. once the user selects the regions to be restored, the algorithmic program can mechanically reliever these regions with data close them. Shenfeng Li presents a replacement image inpainting technique supported exemplar-based imageinpainting plan by Curvature-Driven Diffusion (CDD) model during this paper. This technique improves effectiveness and also the linear structure propagation by rational confidence and information computing technique. Therefore, the strategy projected will effectively forestall the 
"garbage" from manufacturing throughout the method of inpainting, that may be a common drawback featured in different ways

\section{IMPLEMENTATION}

\subsection{Block Diagram}

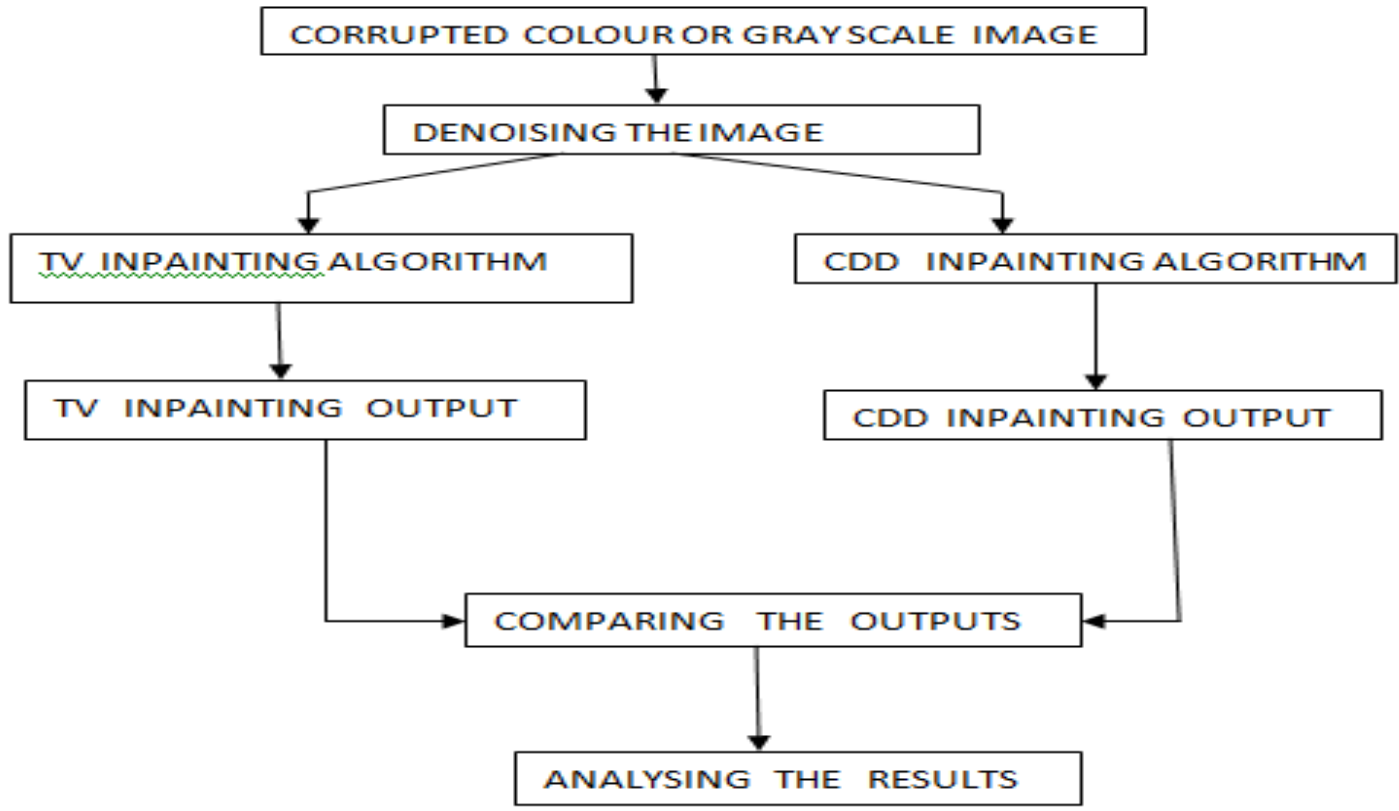

Fig 1. Flow of method to be implemented

- Image with painted knowledge has got to be removed with use of 2 formula i.e CDD inpainting and $\mathrm{TV}$ ( Total variation) image inpainting.

- The 2 inpainting techniques to use are:

\section{1) CDD: Curvature driven diffusion}

It is supported the answer of partial equation of isophote intensity to relief missing parts within the region into account.

\section{2)TV:Total Variation}

It is supported texture inpainting. The filling-in method is mechanically exhausted regions containing fully completely different structures, textures, and encompassing backgrounds.

- We have to be compelled to compare their Signal to noise quantitative relation

\subsection{Inpainting via CDD inpainting:}

The cdd model refers to,

$\delta \mathrm{u}($ or 0$)=\mathrm{g}(|\mathrm{k}|) \quad \mathrm{u} \quad, \mathrm{x} \varepsilon \mathrm{D}$

$\delta \mathrm{t}|\mathrm{u}| \mathrm{u}=\mathrm{u} 0 \quad, \quad \mathrm{x} \varepsilon \mathrm{Dc}$

Here the inpainting domain D is mathematically thought of as associate open set, ie not as well as its boundary ; and $\mathrm{u}$ is accessible a part of the image. If we tend to solve the time walking equation ,then the initial condition are often any compatible guess, that is any $\mathrm{u}(\mathrm{x}, 0)$ that satisfies $\mathrm{u}(\mathrm{x}, 0)=\mathrm{u} 0(\mathrm{x}), \mathrm{x} \in \mathrm{Dc}$

The flux field for the curvature -driven diffusion is,

$$
\mathrm{j}=-\mathrm{D} \mathrm{u}=-(\mathrm{g}(|\mathrm{k}|) /|\mathrm{u}|) \mathrm{u},
$$

Which is opposed gradient and thence stable . Physically we will treat the image operate $\mathrm{u}$ as density operate of bound kind of particles. The on the market a part of original image $\mathrm{u} 0$ acts as a relentless supply or sink of particles. for instance, suppose we tend to area unit inpainting a broken bar in uniform background .

The connecting of 2 broken components is accomplished, in this particle diffusion image, by the particle perpetually fluxed into the inpainting domain through boundaries. 


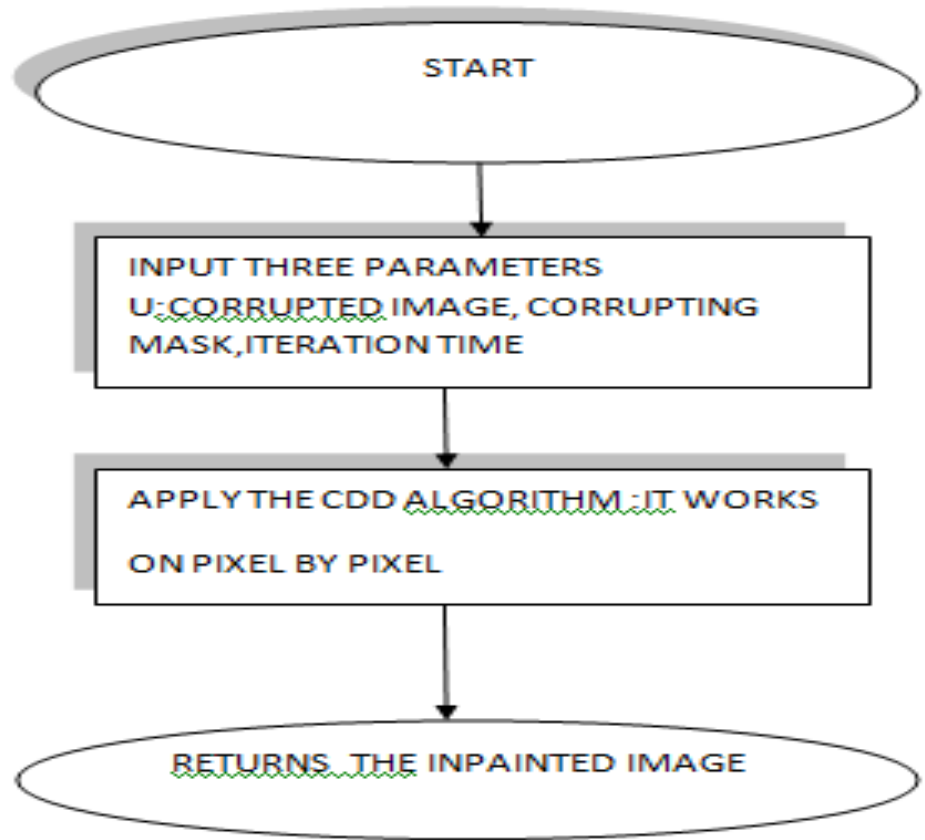

\subsection{Analysis methodology to use for TV inpainting:}

- Design a operate to solves the entire Variation inpainting downside $\min \mathrm{TV}(\mathrm{X})$ subject to $\| \mathrm{X}(\mathrm{Ic})$ - $\mathrm{B}(\mathrm{Ic}) \| \_\mathrm{F} \& \mathrm{lt} ;=$ delta

where $\mathrm{B}$ may be a screaming image with missing pixels, Ic area unit the indices to the intact pixels, $\mathrm{X}$ is that the reconstruction, associated delta is an upperbound for the residual issue. The TV operate is that the 1-norm(factor)of the gradient magnitude, computed with facilitate of neighbourpel variations. At the image borders, we tend to obligatory reflexive boundary conditions for the gradient computations.

- The info relating to the intact and missing pixels is given within the kind of the mask $\mathrm{M}$ that may be a matrix of constant size as $\mathrm{B}$, and whose nonzero parts indicate missing pixels.

- The parameter delta ought to be of constant size because the norm of the image noise. If the image is mtimes-n, and alphabetic character is that the variance of the image noise in an exceedingly pel, then we tend to advocate to use delta $=\operatorname{tau}^{*} \operatorname{sqrt}(m * n) *$ sigma, wherever letter of the alphabet is slightly smaller than one, say, tau $=0.85$.

- The operate should come back associate epsilon-optimal resolution $X$, that means that if $X^{*}$ is that the precise resolution, then our resolution $X$ satisfies $\operatorname{TV}(X)-\operatorname{TV}\left(X^{*}\right)$ \&lt; = letter of the alphabet $=$ $\max (B(I c)) * m * n * e p s \_r e l$, wherever eps_rel is that the fixed relative accuracy; the default is eps_rel $=1 \mathrm{e}-3$. 


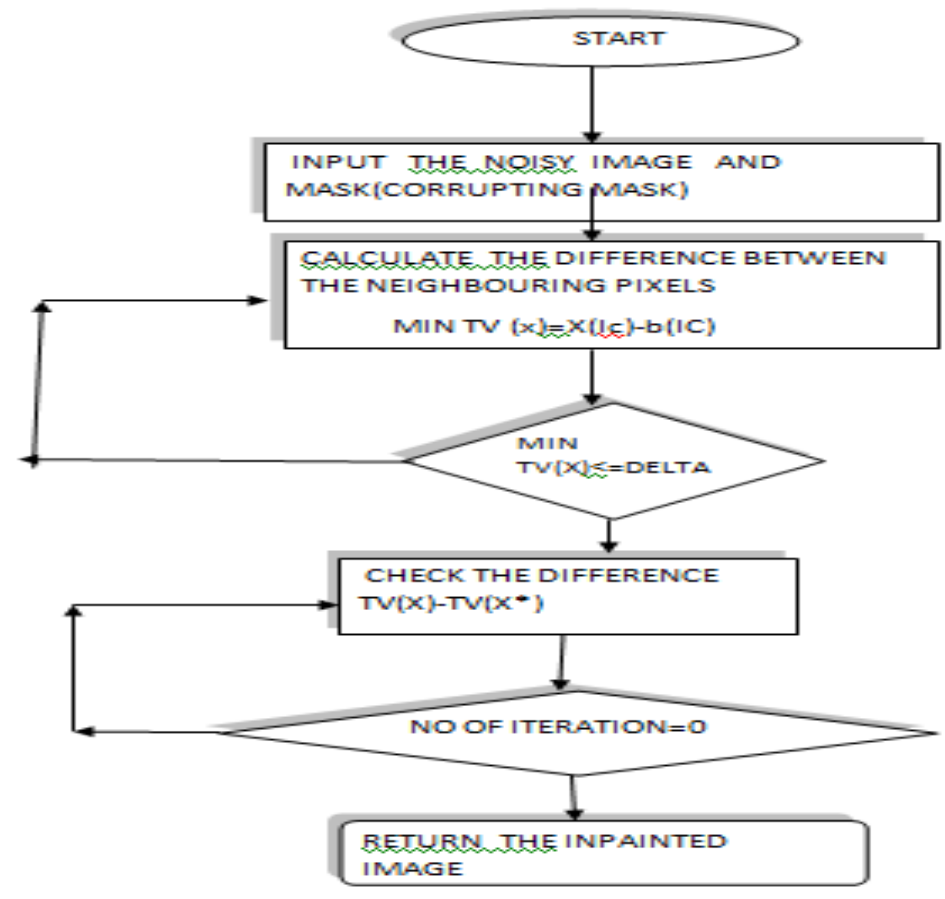

IV. FUTURE WORK

Proposed algorithmic rule has got to be hardware enforced i.e the high-density lipoprotein writing of algorithmic rule has got to be written using VHDL/ Verilog and generating RTL of planned style. Implement the algorithmic rule in FPGA. style has got to be through with low power and low RTL needed. High outturn has got to be achieved by victimization high outturn core i.e Virtex6 or Cyclone -II core.

\section{Output of CDD And TV Inpainting}

\section{RESULTS}

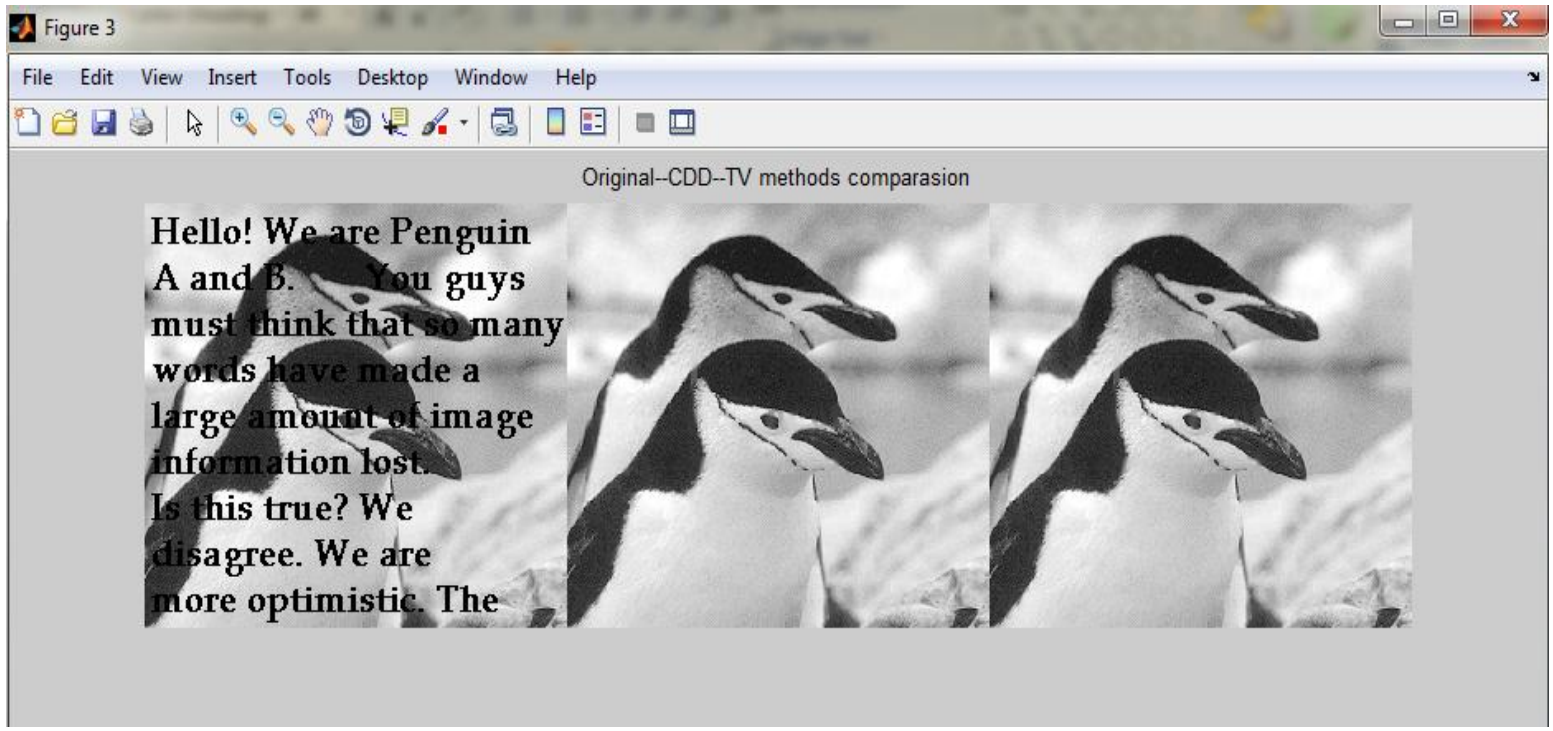



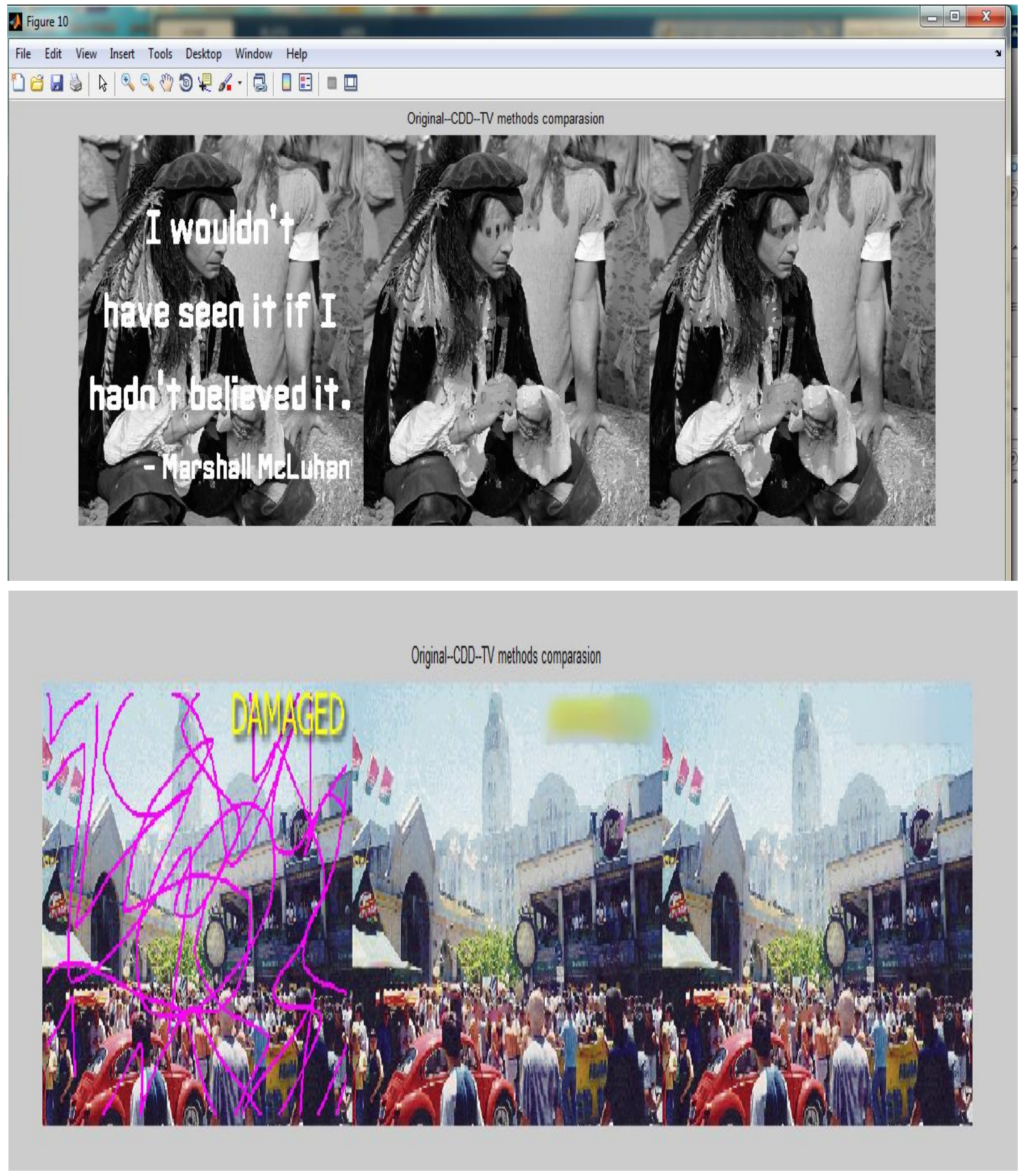

\section{CONCLUSION}

In this paper two algorithms of inpainting square measure is implemented:

(1) CDD(Curvature Driven Diffusion)

(2) TV(Total Variation)

The choice of victimization CDD and television algorithms with super resolution depends on the character of the image to be inpainted.

The time needed for the inpainting method depends on the dimensions of the image and therefore the regions to be inpainted, and it ranges type few seconds to many minutes for big pictures. The time analysis are undertaken.

Some results are shown.Our developed algorithmic rule ought to reproduce texture and at a similar time keep the structure of the encircling space of the inpainted region. 


\section{REFERENCES}

[1] Olivier Le Meur ,Christine Guillemot,: Super resolution based inpaintingIEEE Trans. OnImageprocessing vol.pp no. 992013

[2] Bugeau, A., Bertalm'10, M., Caselles, V., Sapiro, G.: A comprehensive frameworkfor image inpainting. IEEE Trans. on Image Processing 19 (2010)2634-2644

[3] Glasner, D., Bagon, S., Irani, M.: Super-resolution from a single image. In: In 2009IEEE 12th International Conference on Computer Vision (ICCV). Volume 10.(2009)349356

[4] Minqin Wang: .TV-Based Texture Image Inpainting:In: International Conference onMultiMedia and Information Technology.(2008)272-275

[5] Criminisi, A., P'erez, P., Toyama, K.: Region filling and object removal by examplar-based image inpainting IEEE Trans. On Image Processing 13 (2004)1200-1212

[6] Drori, I., Cohen-Or, D., Yeshurun, H.: Fragment-based image completionACM Trans. Graph.2 (2003)303-312

[7] Freeman,W.T., Jones, T.R., Pasztor, E.C.: Example-based super-resolution. IEEEComputer Graphics and Applications 22 (2002)56-65

[8] Chan T., Shen J.: Variational restoration of non-flat image features: models andalgorithms. SIAM J. Appl. Math. 61 (2001)1338-1361

[9] Efros, A.A., Freeman, W.T.: Image quilting for texture synthesis and transfer. In:SIGGRAPH. (2001)341-346

[10] Bertalmio, M., Sapiro, G., Caselles, V., Ballester, C.: Image inpainting. In: SIG-GRPAH 2000. (2000)

[11] Efros, A.A., Leung, T.K.: Texture synthesis by non-parametric sampling. In: International Conference on Computer Vision. (1999) 